\title{
Strain and strain rate parametric imaging. A new method for post processing to 3-/4-dimensional images from three standard apical planes. Preliminary data on feasibility, artefact and regional dyssynergy visualisation
}

\author{
Asbjørn Støylen*, Charlotte B Ingul and Hans Torp
}

Address: Department of Circulation and Medical Imaging, Faculty of Medicine, Norwegian University of Science and Technology, Trondheim, Norway

Email: Asbjørn Støylen* - a-stoe@online.no; Charlotte B Ingul - charlotte.B.Ingul@medisin.ntnu.no; Hans Torp - hans.torp@medisin.ntnu.no

* Corresponding author

Published: 25 August 2003

Cardiovascular Ultrasound 2003, I:I I
Received: 25 June 2003

Accepted: 25 August 2003

This article is available from: http://www.cardiovascularultrasound.com/content/l/I/II

(C) 2003 Støylen et al; licensee BioMed Central Ltd. This is an Open Access article: verbatim copying and redistribution of this article are permitted in all media for any purpose, provided this notice is preserved along with the article's original URL.

\begin{abstract}
Background: We describe a method for 3-/4D reconstruction of tissue Doppler data from three standard apical planes, post processing to derived data of strain rate / strain and parametric colour imaging of the data. The data can be displayed as M-mode arrays from all six walls, Bull's eye projection and a 3D surface figure that can be scrolled and rotated. Numerical data and waveforms can be re-extracted.
\end{abstract}

Methods: Feasibility was tested by Strain Rate Imaging in 6 normal subjects and 6 patients with acute myocardial infarction. Reverberation artefacts and dyssynergy was identified by colour images. End systolic strain, peak systolic and mid systolic strain rate were measured.

Results: Infarcts were visualised in all patients by colour imaging of mid systolic strain rate, end systolic strain and post systolic shortening by strain rate. Reverberation artefacts were visible in 3 of 6 normals, and 2 of 6 patients, and were identified both on bull's eye and M-mode display, but influenced quantitative measurement. Peak systolic strain rate was in controls minimum -I.II, maximum -0.89 and in patients minimum -1.66 , maximum $0.02(p=0.04)$. Mid systolic strain rate and end systolic strain did not separate the groups significantly.

Conclusion: 3-/4D reconstruction and colour display is feasible, allowing quick visual identification of infarcts and artefacts, as well as extension of area of post systolic shortening. Strain rate is better suited to colour parametric display than strain.

\section{Background}

\section{Parametric imaging}

Colour tissue Doppler [1] samples tissue velocities, nearly simultaneously from all pixels in the 2D picture. Data are quantitative, and can be displayed as curves or numbers. Quantitative analysis in all segments of the left ventricle, however, is time-consuming. Colour display, or paramet- ric imaging, reduces the displayed data to semi-quantitative information, allowing a quick visual assessment of functional data over a larger area. In addition, this display allows new measurements, such as area and propagation velocity. 


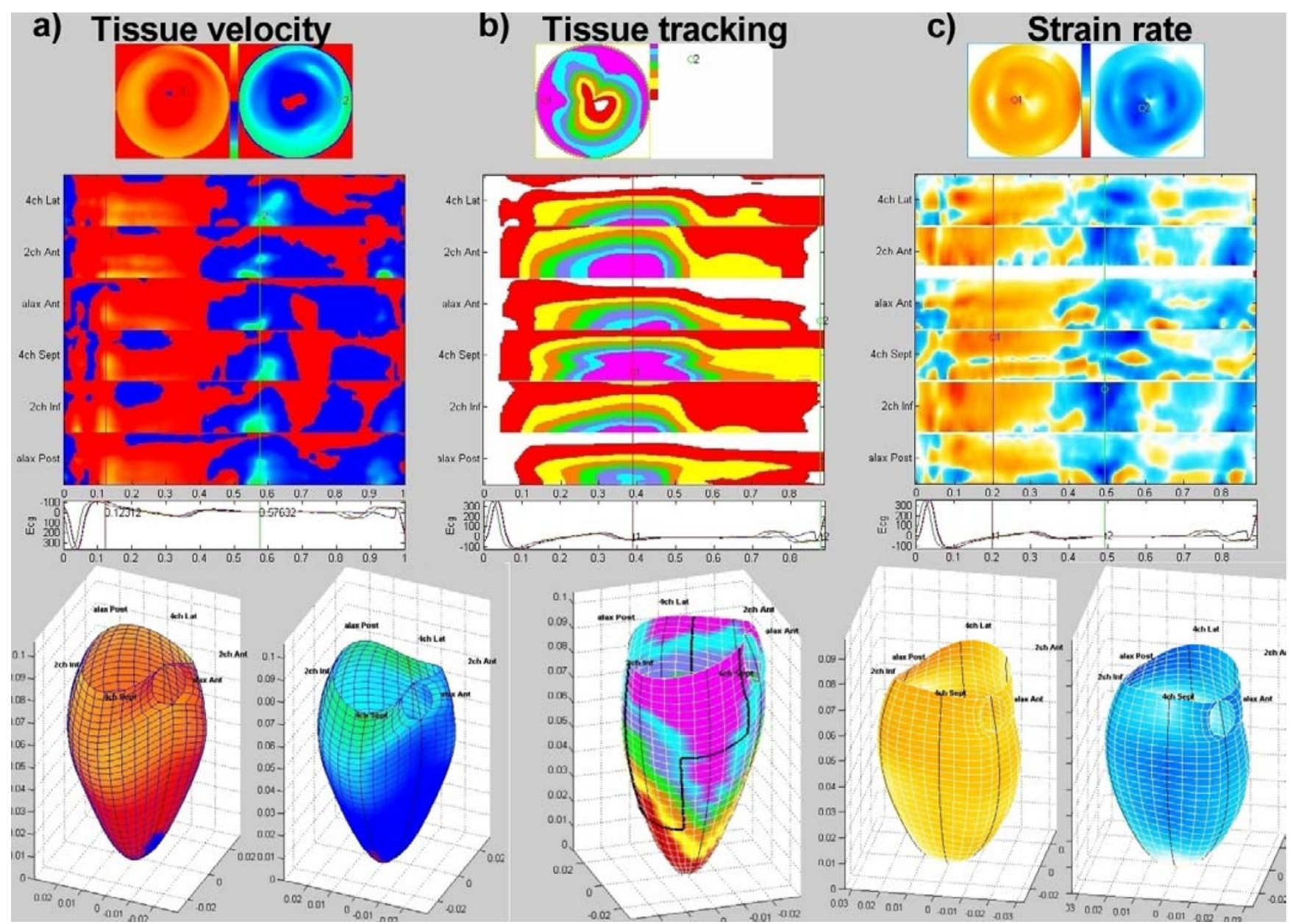

Figure I

Different parametric imaging in 3-/4D display, all from a normal subject. Left to right: The red-blue display of tissue velocity, the coloured bands of tissue tracking and the yellow-blue display of strain rate. In tissue velocity, lighter colour represents higher velocities, showing clearly the velocity gradient from base to apex both in systole and diastole. In tissue tracking, each colour represents an interval of $2 \mathrm{~mm}$ displacement, as shown by the legend. This means that red represents $2-4 \mathrm{~mm}$ displacement, increasing to magenta showing $>14 \mathrm{~mm}$ at the base. Strain rate shows shortening in yellow to red, lengthening in blue, darker colour represents more deformation. Some inhomogeneity is visible due to noise and dropouts. Top to bottom: Bull's eye display both in systole and diastole (except tissue tracking), M-mode array from all six walls with apex on top and base at the bottom with ECG and a 3D surface reconstruction, velocity and strain rate in systole and diastole. The bull's eye projection shows all of the surface, but the area is distorted; the apex is progressively diminished, while the base is over-represented, the 3D figure shows a representation of the true area, but has to be rotated to se all of the surface. Reconstruction is done from three separate cine-loops, synchronised by means of ECG. The ECG at the left is inverted, but is from the same patient, as may be seen by the end of the cycle, where there is noise in the ECG signal. The aortic annulus and location of the imaging planes are added for orientation.

In colour display, velocities toward the transducer are shown in red, away from the transducer in blue (fig. 1a). Tissue velocity is especially sensitive in showing regional asynchrony.

Velocity data, being simultaneous and digital, can be post processed to derived parameters. Numerical integration of velocity over time, results in displacement curves, reproducing the motion curves from M-mode. Both velocity and displacement are imaging of local motion. Tissue tracking, an imaging modality developed at the Karolinska Institutet in Stockholm [2], displays displacement by bands of different colours (fig. 1b). In this modality global function is visualised by the annular displacement, 
which is related to EF [3-5], local motion by the colour, regional differences by the symmetry and deformation by the width of the bands. Tissue tracking is thus the only parametric imaging modality that displays both motion and deformation at the same time.

Strain rate imaging, a modality developed at the Norwegian University of Science and Technology in Trondheim [6], is a spatial derivation of velocity: $S R=((v(x)-v(x+$ $\Delta \mathrm{x})) / \Delta \mathrm{x}$ in the direction of the ultrasound beam. This algorithm subtracts regional velocities due to translation and tethering, giving in the rate of regional deformation strain rate, an index of regional contractility [7]. Clinical findings of reduced regional function in myocardial infarction have been validated against 2D echocardiography [8] and coronary angiography [9]. In strain rate, yellow to orange represents shortening, cyan to blue lengthening (fig 1c). The numerical derivation results in a less favourable signal - to - noise ratio in strain rate than in velocity $[9,10]$, resulting in increased random noise. Strain - local deformation - can be measured by temporal integration of strain rate. Strain measurements by this method have been validated against ultrasonomicrometry [11] and MR [12]. The integration process eliminates most of the random noise, but strain is still sensitive to non-random noise, and in addition prone to baseline drift. Both strain and strain rate are deformation imaging, as opposed to motion, and are sensitive for regional ischemia. They are translation and tethering independent, as opposed to motion, and will thus show the true location of regional dyssynergy $[6,8,9]$. On the other hand, experience with the method is necessary for discerning true pathology from artefacts due to dropouts and reverberations [9].

\section{Display}

Various display options are available, depending on the kind of information desired (figs. 1 and 2). Curved anatomical M-mode [13] shows the whole time sequence, in one wall at a time. This displays space - time relations $[14,15]$. Bull's eye projection is a 2-dimensional map of the entire surface of the left ventricle, but only at one instance in time. Bull's eye display is common in SPECT imaging and in regional wall motion display in echocardiography [16].

We present a method where the velocity data from 3 standard apical imaging planes are acquired, integrating spatial information and the temporal sequence results in a four-dimensional data set. From this, curved M-modes, bull's eye views and a 3-dimensional surface figure can be generated. The four-dimensional nature of the data results in the ability of the display to be scrolled through the heart cycle. The method also includes the post processing ability to derive any secondary parameter such as motion, strain rate and strain and the ability to re-extract numeri- cal data as curves or numbers. The basic principles are illustrated in fig. 2 .

The aim of this study was to evaluate the feasibility of this imaging modality in a clinical setting, to display regional dyssynergy and artefacts and to see if regions of maximum and minimum contractility could be identified for quantitative measurement.

\section{Methods \\ Patients}

Six patients with first, acute myocardial infarction, two inferior, four anterior, were examined. The patients participated in a prospective study of strain rate in myocardial infarction, and examinations were done during the first two days. Six normal subjects were examined as controls. These participated in a stress echo study, selected on the basis of a normal coronary angiography done for clinical reasons, and all had normal resting echocardiogram by conventional criteria. Resting cine-loops were used for the present study. All studies were approved by the regional ethical committee, and written consent was obtained. The patients were included consecutively, and no patients excluded for poor image quality.

\section{Echocardiography and post processing}

Echocardiography was performed with a GE Vingmed Vivid 7 scanner (GE Vingmed Ultrasound, Horten, Norway), with tissue Doppler acquired at a frame rate of 100 - 150 FPS. Single cine-loops from each standard apical plane - 4-chamber, 2-chamber and apical long axis - were transferred to a PC computer for post processing in experimental software (GcMat, GE Vingmed Ultrasound), programmed in Matlab (MathWorks inc., USA).

First, a curved M-mode was drawn in each of the three planes by placing seven points and curve drawn automatically by spline interpolation. Velocity data were sampled along the line. Temporal smoothing over 3 frames, and spatial smoothing over 3 pixels was applied automatically. Strain rate and strain was processed with an offset length of $12 \mathrm{~mm}$. Adjustment for unequal RR-intervals was achieved by resampling of 200 points of the ECG curve, and the velocity data interpolated to fit the ECG curves. For rotational angle between planes, an assumption of $60^{\circ}$ between the planes (being the average separation) was applied in the program, and velocity data were interpolated by cubic spline by a method described previously [17]. Data was then displayed as bull's eye (fig. 1, top), curved M-mode array (fig. 1, middle) and 3-dimensional surface grid (fig. 1, bottom). In 3D display, the imaging planes and the aortic annulus were added for orientation. The whole of this post processing took $2-3$ minutes. 

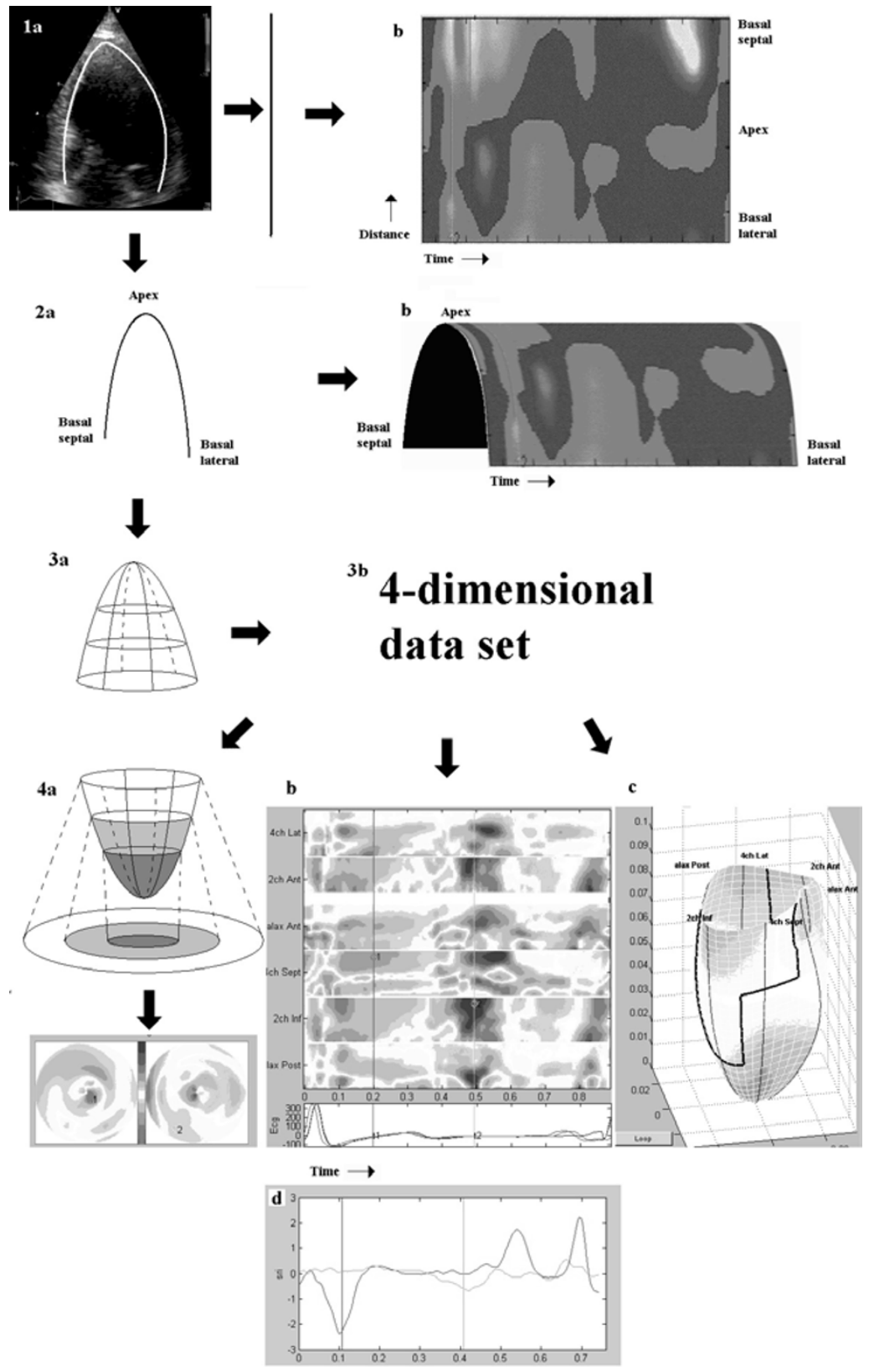

Figure 2

Illustration of the four-dimensional data set. Drawing a curved M-mode along the wall (Ia) in one plane, results in velocity data along a straight line. As data are recorded over a time period, adding the time sequence in each pixel of the line, results in a traditional curved M-mode plot. This is equivalent to a 2-dimensional surface (Ib) with one spatial and one temporal dimension. However, the curved M-mode contains more information; the curvature itself defines the location of each point in space relative to all the others. With this information added, the M-mode is a curve in a plane, i.e. a two-dimensional figure (2a) instead of a straight line. If the time-sequence is added to this figure, analogous to the transition Ic, it results in a curved plane, a three-dimensional figure, with two spatial and one temporal dimension (2b). This kind of display is not used in practice; it is included here for reasons of analogy only. Adding two more curved M-modes from the two other standard planes and information about their angular separation, results in a three-dimensional figure, a grid (3a) the spatial interpolation is done by cubic spline. When the time-sequence is added in this case, the data set becomes four-dimensional (3b). This cannot be shown directly, but is illustrated by the analogy to $I$ and $2 b$. Only part of the information can be extracted at a time, for the various display modalities: Bull's eye at any point in the heart cycle (4a), M-mode array (4b), 3D surface (4c). Only the processing to the full data set, however, allows the figures to be scrolled through the heart cycle, and the data set remains quantitative, allowing the curves to be extracted from each point. 


\section{Analysis}

Bull's eye / M-mode array and 3D surfaces as shown in fig. 3 , were computed for each subject. The bull's eye was evaluated visually in mid systole for homogeneity of strain rate, in end systole for homogeneity of strain and in early diastole for visible presence of post systolic shortening in strain rate. Post systole was identified as the period after the T-wave in ECG, before and during early filling phase identified in strain rate [14].

The points of maximum a minimum strain rate in mid systole were identified visually, and mid systolic and peak systolic strain rate as well as end systolic strain were measured in these points. Care was taken not to avoid the areas of reverberations or dropouts, as the object was to evaluate the impact of artefacts in this feasibility study. The differences between maximum and minimum strain rate was calculated.

\section{Statistics}

No statistical analysis was applied to the visual assessment; all data are displayed in fig. 4. For quantitative analysis, test for difference between the groups are by twotailed student's T-test of unequal variance.

\section{Results Qualitative analysis}

Bull's eyes from all subjects are shown in fig. 4. As can be shown, mid systolic strain rate was reasonably homogenous in four normal subjects, the other two showing large reverberations, and in the case of no 6, large dropouts as well. In end systolic strain, only three out of six were homogenous Reverberations are clearly visible as a ringshaped figure in bull's eye and horizontal bands of inverted colour in the M-modes (fig. 5). In addition, there were inverted strain colour in the base, both in controls and patients.

Infarcts were visible as inhomogeneous mid systolic strain rate and end systolic strain; both parameters located the infarcts equally well. Post-systolic shortening was evident in early diastolic strain rate in all patients. The area with post-systolic shortening was visually larger than the area of dys- to hypokinesia in mid systole. Post-systolic shortening could not be identified reliably in colour imaging, even when present in the curves. Reverberations were present in one patient, basal artefact in one, both in strain rate and strain.

\section{Quantitative analysis}

Mid systolic and peak systolic strain rate as well as end systolic strain values are given in table 1 . It is evident, that in quantitative analysis, only peak systolic strain rate showed difference between normals and myocardial infarction patients.
Neither the trend toward hypokinesia of the infarcted areas by mid systolic strain rate and end-systolic strain, nor the trend toward hyperkinesia in non-infarcted segments in patients, was significant. Reverberations could not be reliably identified from curve analysis alone (fig 5 ).

\section{Discussion \\ Basic limitations of strain rate and strain imaging}

It must be emphasised that the present study is about the method of 3-dimensional reconstruction and display, while the basic method of strain rate imaging is not different from that published previously $[6-12,14]$. Strain rate imaging in itself is based on post processing, and has a number of inherent limitations.

The most basic limitation is the one-dimensional nature of the data. While deformation is three-dimensional (longitudinal, transverse and circumferential), the present tissue Doppler derived method measures only longitudinal deformation. However, heart muscle is generally assumed incompressible, which means conservation of volume. Hence, strain in three dimensions must balance (the basic equation in volume conservation: $\varepsilon_{\mathrm{x}}+\varepsilon_{\mathrm{y}}+\varepsilon_{\mathrm{z}}=0$ ), so in systole longitudinal plus circumferential shortening equals transverse thickening, and vice versa in diastole. Previous studies have shown similar diagnostic information by longitudinal strain rate and transverse wall thickening (which is the same as transverse strain) by ordinary echocardiography [9]. This means that the longitudinal strain rate may be representative of the compound regional deformation. The practical value for clinical work of adding more deformation directions remains to be demonstrated.

Data represents only a line along the wall, but to achieve a high frame rate, the tissue Doppler beams have to be wide, and the lateral resolution is low anyway. This means that differential strain across the wall cannot be measured, however, this is true for all tissue Doppler derived data sets.

The one-dimensional nature of the data also results in angle distortion, when the ultrasound beam deviates from the direction of the motion. While velocity measurements decrease by the cosine of the insonation angle, strain rate decreases more, adding the transverse strain rate with the opposite value $[6,18]$. This means that in areas where the angle between the wall and the ultrasound beam is more than 45 degrees, the strain rate values and hence, the colour will reverse $[11,18]$. This problem is apparent in the apex and the base [9]. However, all clinical studies of strain rate have found it possible to measure strain rate and strain in the apical segments, by measuring in the basal parts where the walls are more parallel to the ultrasound beams $[8,9,12,14,19,20]$. As can be seen from fig. 

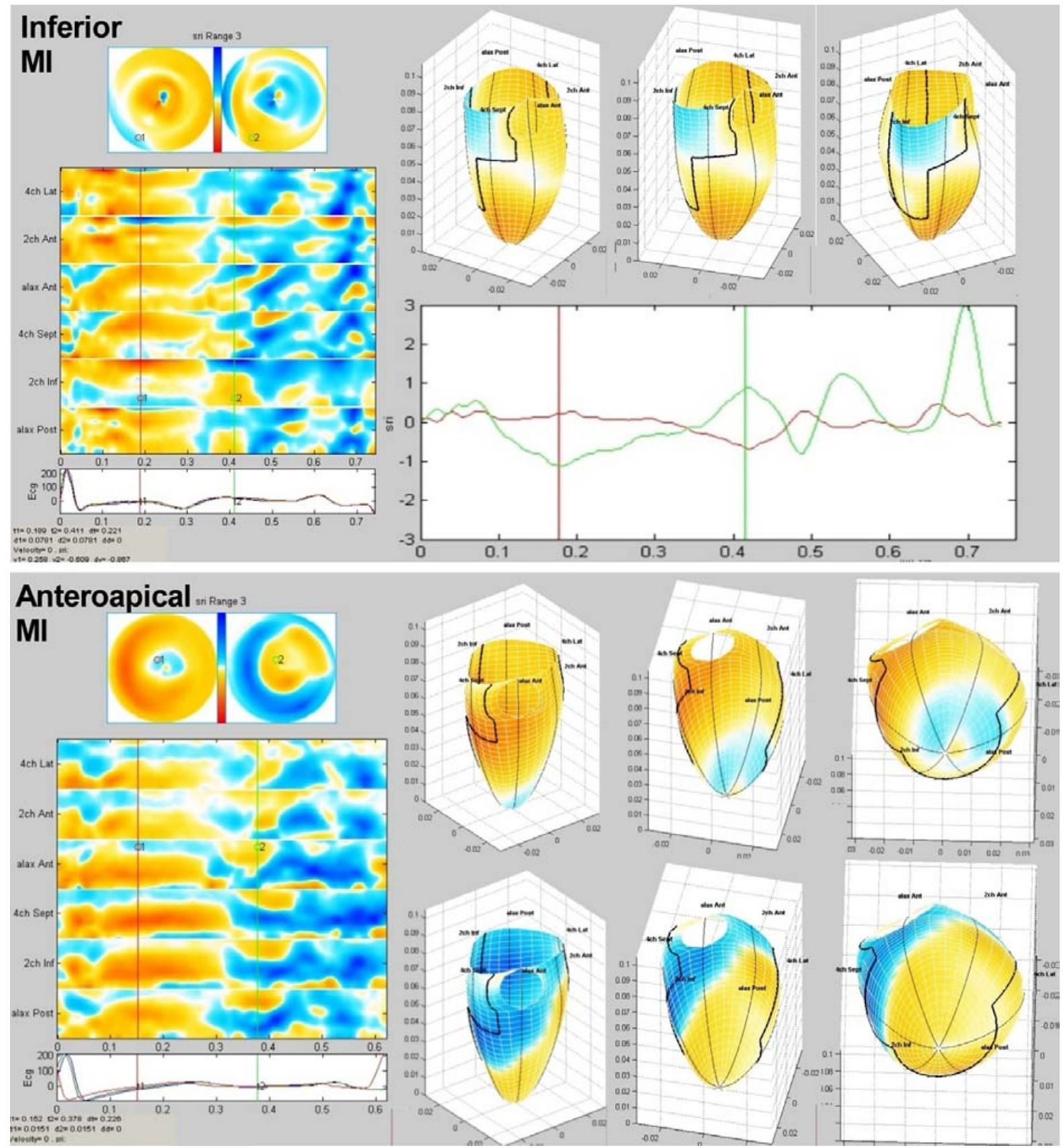

\section{Figure 3}

Strain rate examples from two patients with myocardial infarction. Bull's eye, M-mode array and 3D surface display. Top half, inferior infarction (patient 2), showing slight systolic dyskinesia to hypokinesia - blue to light yellow - in the basal inferior and inferoseptal segments, hypokinesia in the inferior midwall. Post systolic shortening is shown over a larger area in the same region, visible on bull's eye (2) and M-mode. The 3D display is shown in mid-systole, the three frames illustrates the application's ability to rotate the display, here counter clockwise around the longitudinal axis. In addition is shown the strain rate curves from the infarct in red, demonstrating akinesia and post systolic shortening, and from a normal segment in green, showing normal systolic shortening with peak systolic strain rate of $<-\mathrm{I} \mathrm{s}^{-1}$, and diastolic lengthening. Bottom half shows an anteroapical infarct (patient 6), with a small area of dyskinesia, surrounded by a larger area of hypokinesia. (A very small area of inverted colour due to angular distortion can be seen.) Again, the area of post systolic shortening is in the same region, but larger, the difference probably being the ischemic border zone. The 3D reconstruction, systole in the upper row, and early diastole in the lower row, is rotated from an anterior to an apical view. Faulty ECG trigging on the scanner can be seen by the ECG curves on this patient, showing that the reconstruction must be inhomogenous. 

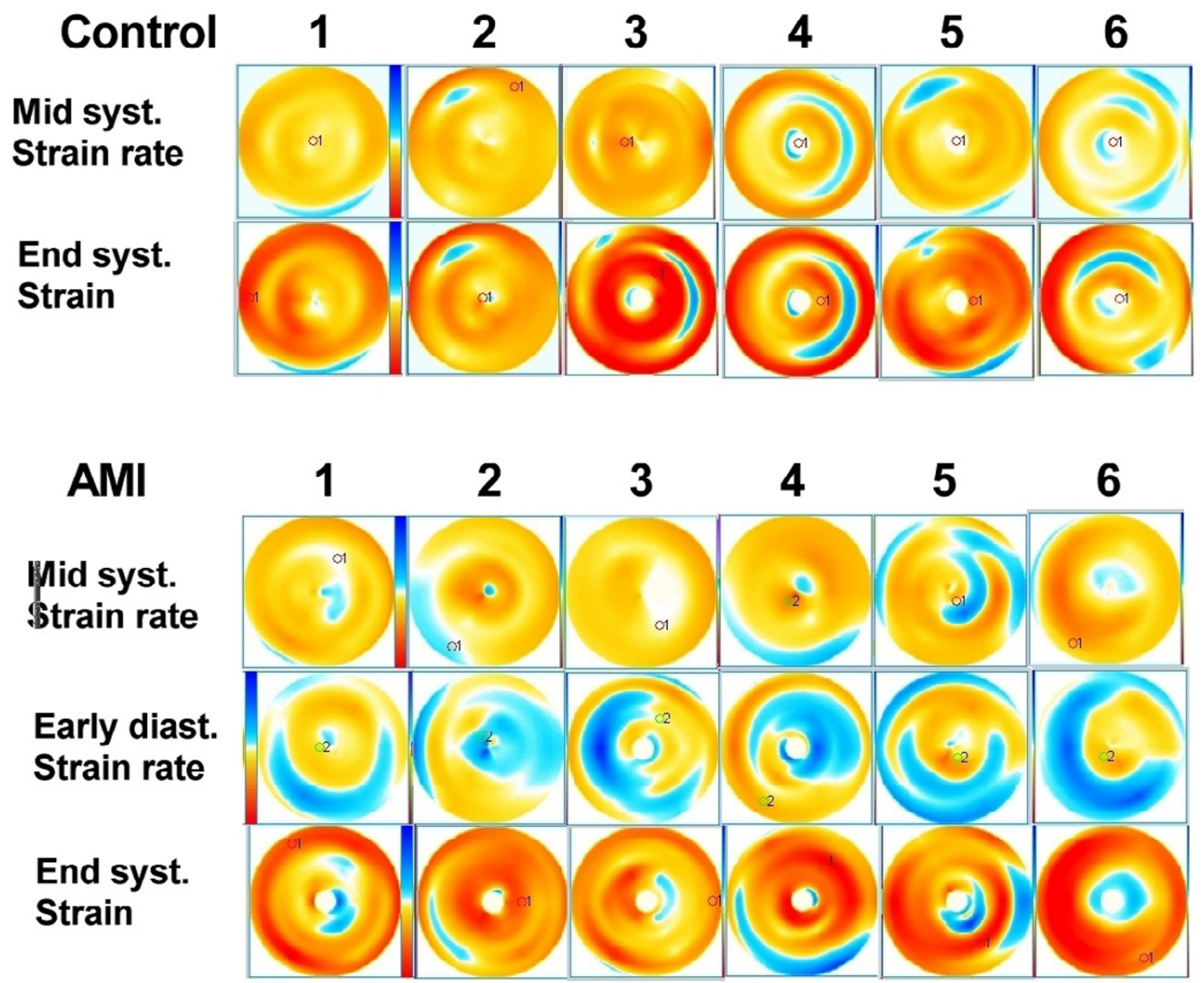

\section{Figure 4}

Bull's eye views of all subjects in the study. The colour legends are reproduced, with yellow to red being shortening, blue being lengthening, and deeper colour means greater magnitude of either shortening or lengthening. The same colour legend is used for strain rate and strain, but the scales differ somewhat, resulting deeper colour in the strain images. The two upper rows are the normal subjects, mid systolic strain rate and end systolic strain. In control subjects 2 and 5 a small defect top left is seen, which is the aortic root. Large reverberations are seen in strain rate and strain in subject 4 and 6 , and in strain in subject 3 as well. Reverberations are seen as circular bands at the midwall level, being due to the spatial interpolation process. In addition, basal artefacts are seen in subject I, 5 and 6. Subject 6 shows areas of light colours due to dropouts as well. The three bottom rows are the infarction patients. Patients 2 and 4 had inferior, the rest apical infarctions. The infarcts have a more typical location, but the infarct in patient 4 may be difficult to separate from the basal artefacts in control subjects. In patient 5 , there are reverberation artefacts as well, seen in the lateral base. The diagnosis of infarction was as easy with mid systolic strain rate as with end systolic strain, as is apparent in this figure. However, by adding post systolic shortening, the diagnosis of infarction was facilitated. Post systolic shortening was present in all six patients in this acute phase, and is seen to extend beyond the area of hypo- and dyskinesia in all. In the early diastolic images, there is visible inhomogeneity that is a normal phenomenon, not the results of pathology or reverberations. In some subjects small areas of inverted colour in the apex due to angular distortion can be seen, however, this area is small, and in most images it has been blanked. 


\section{Normal AMI Reverb.}

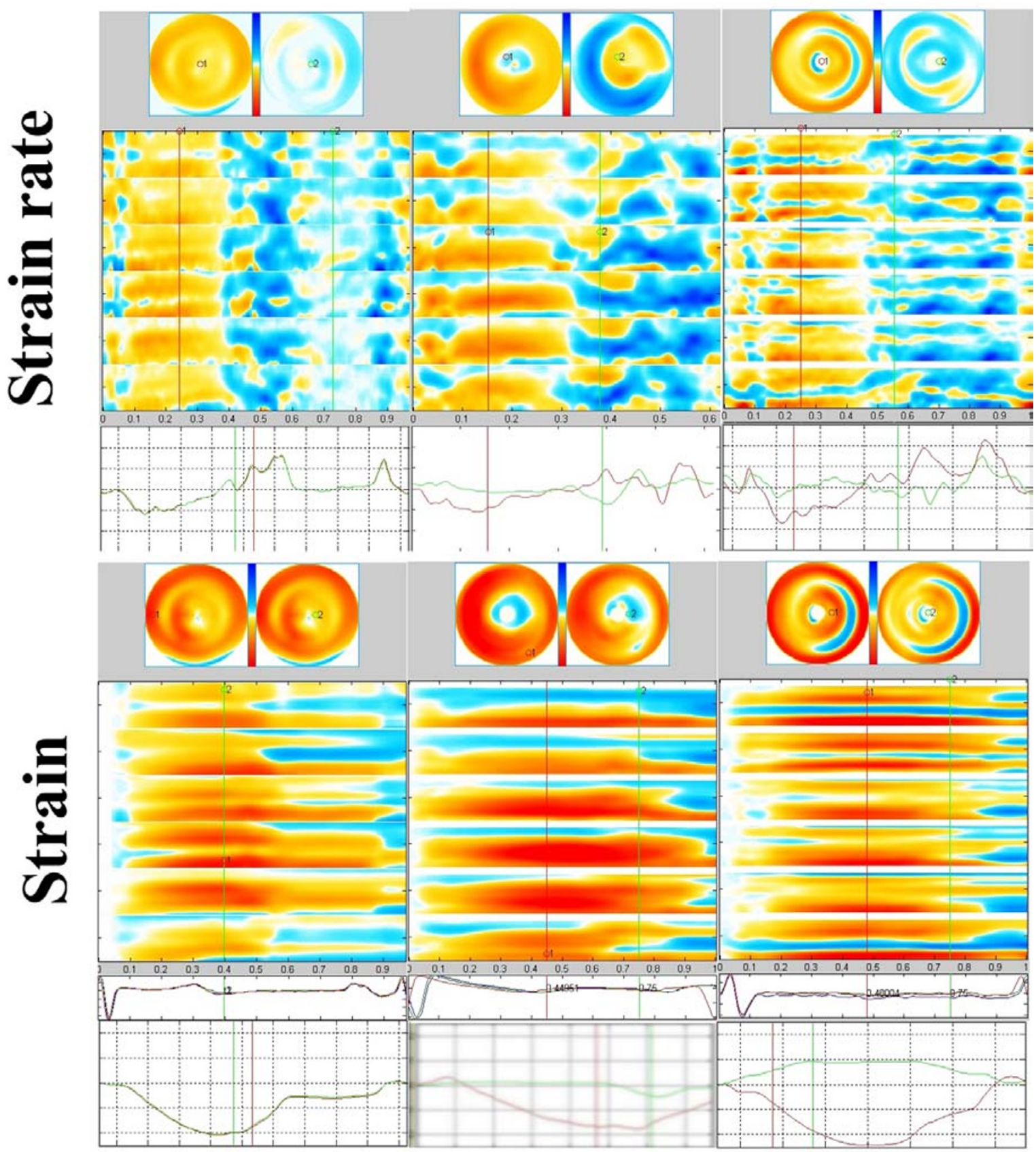

Figure 5

The appearance of reverberations versus normal and pathological findings. Left to right: normal subject with good quality images (control I), patient with anteroapical infarction (patient 6) and normal subject with poor image quality (control 4). Top to bottom: Strain rate bull's eyes, M-mode arrays and curves, Strain bull's eyes M-mode arrays, ECG and curves. The red colour of strain is seen to extend well into diastole normally, so post systolic shortening in pathology cannot be discerned by colour strain images. In the infarct, apical hypo- to akinesia is visible in all modalities, and post systolic shortening in colour strain rate and both strain rate and strain curves. Reverberations are seen as midwall circular lines of inverted colour in the bull's eye, and as horizontal lines of inverted colour in the M-modes. In strain rate curve it may give the impression of akinesia with post-systolic shortening (although not quite typical), but the horizontal lines of reverberations in M-mode array is typical. In strain, findings are typical reverberations, also in the curve. 
Table I: Average maximum and minimum mid systolic and peak systolic strain rate and end systolic strain.

\begin{tabular}{|c|c|c|c|c|c|c|c|c|c|}
\hline & \multicolumn{3}{|c|}{ Mid syst. strain rate $\left(\mathrm{s}^{-1}\right)$} & \multicolumn{3}{|c|}{ Peak syst. strain rate $\left(\mathrm{s}^{-1}\right)$} & \multicolumn{3}{|c|}{ End syst. strain (\%) } \\
\hline & Max. & Min. & Diff. & Max. & Min. & Diff. & Max. & Min. & Diff. \\
\hline Controls: & $-0.01(0.16)$ & $-1.21(0.27)$ & $-1.20(0.35)$ & $-0.89(0.77)$ & $-1.11(0.70)$ & $-0,21(1.31)$ & $0 \%(3)$ & $-22 \%(3)$ & $-22 \%(5)$ \\
\hline Patients: & $0.05(0.17)$ & $-1.51(0.37)$ & $-1.36(0.39)$ & $0.02(0.16)$ & $-1.66(0.36)$ & $-1.68(0.36)$ & $5 \%(7)$ & $-25 \%(4)$ & $-30 \%(9)$ \\
\hline P: & NS & NS & NS & 0.03 & NS & 0.04 & NS & NS & NS \\
\hline
\end{tabular}

Measurements made in points identified as max and min. mid systolic strain by bull's eye (standard deviations in parentheses). In systole, the deformation is shortening, i.e. negative values, so the lowest negative value indicates most deformation, i.e. highest contractility.

4, the area of distortion is quite small, and during the last part of the study, blanking was applied to this area to avoid displaying misleading information. This limitation is more theoretical than practical.

Strain rate / Strain imaging are prone to reverberation artefacts, due to the basic algorithm. If one of the two velocities is measured as zero, the strain rate value will be inverted in part of the band. The $3 \mathrm{D}$ reconstruction does not add to this, but makes it visible, it is the identification, not the presence of artefacts that are influenced by this method.

Strain rate is sensitive to random noise, and smoothing of curves has to be applied, resulting in loss of temporal resolution. This might lead to underestimation of peak values, but in parametric imaging, being semi-quantitative, this is less important.

\section{Findings}

3D reconstruction is a method for quick visualisation of global distribution of deformation. Infarctions were visible as dyssynergy in the typical anteroapical or inferior locations, and colour display of mid systolic strain rate and end systolic strain were equally sensitive. Post-systolic shortening [21], was an additional finding in all infarcts. Thus, it represents an additional diagnostic criterion, facilitating visual diagnosis of acute infarction. The mechanism for the phenomenon is incompletely understood, but has been clearly documented as a marker of acute ischemia $[21,22]$. It is interesting that the area of postsystolic shortening was larger than the area with hypo- to dyskinesia in all patients. The difference may show an additional area of partly ischemic myocardium, visualising the border zone at risk. This has been visible by curved M-mode alone in previous studies as well $[8,9]$, although not commented on. Post systolic shortening was visible in both strain rate and strain curves, but only in strain rate colour display.

Reverberations were easily identified. On M-mode they did show up as horizontal bands, on the bull's eye as ring shaped structures. The location, shape and temporal sequence differed from the typical vascular areas, and were thus discerned from true pathology, as shown in fig. 5. The artefacts were not so obvious from the curves alone. Basal artefacts are due to either angle distortion or inclusion of part of the mitral ring or pericardium during initial processing. It is important that in three out of six normal subjects, reverberation noise was visible. This high incidence of artefacts has not been reported previously, and may have important implications for the validity and accuracy of quantitative data in clinical practice.

By identifying regions with artefacts, the colour display may aid in avoiding quantitative measurements in those, and thus may also increase the specificity of quantitative strain rate / strain in clinical practice.

\section{Strain rate vs. strain}

Deformation imaging is better in identifying regions of dyssynergy than motion imaging, due to tethering effects. Velocities decrease from base to apex [23] while peak strain rate is the same in all three levels $[10,14,19,20]$.

Peak strain rate was the only quantitative parameter in this study able to identify the infarct areas, while neither mid systolic strain rate nor end systolic / peak strain could do this. This demonstrates a basic limitation of 3D reconstruction. In 3D display, all parts of the ventricle are (in theory), displayed at the same instance of the heart cycle. All previous works in strain rate, however, have concentrated on peak strain rate, but peak strain rate has not been demonstrated to be simultaneous in all parts of the ventricle. Thus, mid-systolic strain rate (or strain rate at any other point in systole) is not equivalent to peak strain rate. Systolic strain is maximal in end systole in normals, and peak strain thus simultaneous. In theory this should be an advantage in 3D reconstruction, but the presence of reverberations eliminated this.

There was no difference between strain and strain rate in the sensitivity to reverberations and dropouts (fig. 4). Smoothing of strain rate data results in reduction in meas- 
ured peak values, but the temporal resolution is still better in colour strain rate than strain, due to the shifts between positive and negative values as evidenced by the visualisation of post-systolic shortening. Thus, strain rate is best suited to colour display.

\section{Limitations and advantages of the processing method and 3D display}

It may be considered a major limitation that the circumferential resolution is limited to three planes, and that most of the information displayed is interpolation. However, the main point of this method is about display of data. The 16-segment model of the left ventricle [24] is the basis of all analysis of regional function in clinical cardiology, wall motion scoring as well as quantitative measurement by velocity, displacement and strain rate / strain imaging. In all these modalities, measurements are taken as representative of a whole segment, resulting in a circumferential resolution of $60^{\circ}$. This is true of any Bull's eye plot, whether of wall motion score or numerical measurements. Reconstruction to Bull's eye alone, does not add more information, but rather displays it in a more accessible form. The method itself, however, is not limited to three planes, but more planes will be non-standard, however. With a gated rotational probe, the number of planes could be increased, but the post processing time would increase proportionately. However, doubling the number of planes, the whole post processing time would still be less than five minutes. The impact of the number of planes has been extensively studied previously [17]. Three planes result in a correct average of volume and surface area, even in asymmetric ventricles. Variability is high, and decreases substantially with an increase to four planes, and little is gained by further increase. Concerning regional dyssynchrony, more planes would increase the exact delineation of the areas. This, however, applies to the whole concept of segmental analysis.

Using three planes, the average angular separation is $60^{\circ}$. This is in accordance with the standard representation of bull's eye, and was chosen for the visual display. The true position of the planes is closer to 0,62 and $101^{\circ}$. These values could be implemented into the model at need, but previous analysis have shown that a variation of $\pm 15^{\circ}$ results in less than $2 \%$ error [17], so the practical advantage of this correction is dubious.

The radial resolution (along the ultrasound beams) is a little more than strain offset length, around $15 \mathrm{~mm}$; i.e. about twice as good as that of the 16-segment model.

Bull's eye and M-mode array could be obtained more directly, without the $3 \mathrm{D}$ reconstruction. The $3 \mathrm{D}$ surface model, however, incorporates the curvature data, which truly adds new information. The main advantage of this is giving an approximate representation of the true area. By subjective assessment, it was an additional help in differentiating infarct areas from basal artefacts in two patients, as it gave a more correct display of the shape and size of the infarct area. The method has further possibilities of true quantitative infarct area measurement, subject to validation studies.

The 3D surface model gives a more intuitive visualisation of the left ventricle, for clinicians unaccustomed to bull's eye or curved M-mode. However, this is also a potential source of pitfalls. The processing method will result in a grid, whether there are tissue data or dropouts. Appropriate blanking of the apical area should be applied to avoid interpretation of angle distortion in the apex as dyskinesia, but as already discussed; this region is far smaller than the extent of the apical segments. Finally, the representation still only shows longitudinal deformation, but as discussed previously this may reflect compound deformation in more than one dimension.

Combining data in a four-dimensional data set results in versatility. The method described may be seen as a method for storing data, where any desired derived parameter and display method can be extracted. Segmental waveform analysis, as well as any kind of parametric display modality as is M-mode, bull's eye and 3D surface is then available. As shown, each type of display contributes to the total information: Bull's eye by showing the whole of the surface, M-mode by showing space - time relations and $3 \mathrm{D}$ surface by displaying the anatomy of the surface and the correct area.

\section{Compensation for HR variability during examination}

With varying HR, the RR-interval will vary too. Systole and diastole varies differently, so a simple linear correction ("stretching") by the end points of the heart cycle is insufficient. A better approximation would be to correct systole and diastole separately, by identifying a point in end-systole, and then fit the two parts of the cycle separately - a three point correction. The present algorithm instead uses a 200-point correction by ECG, so the correction should in theory be correct down to $5 \mathrm{~ms}$, but the use of ECG makes it vulnerable to noisy ECG curves and to variations in automatic trigging. That this approach is less than perfect can be seen in figs 1 and 3 .

\section{Limitations of the study}

The present study is a feasibility study only, with limited numbers, but allowing a more detailed discussion of technical detail and individual findings. The prevalence of artefacts in a normal population, as well as the identification of those in patients, and the resulting diagnostic accuracy should be addressed in larger studies. It is still evident that interpretation of colour images is experience depend- 
ent, and influences reproducibility of diagnosis. Small numbers may also explain lack of significant differences shown in table 1, but findings that need larger numbers for significance have limited clinical value.

In this study, care was taken not to exclude artefacts from the quantitative analysis, to show the impact of those. As the study has demonstrated, colour imaging assists in identifying the artefacts, and the impact of identifying and eliminating artefacts before quantitative analysis has to be studied prospectively.

Post-systolic shortening, or thickening in the transverse direction [23] is generally defined as shortening after closure of the aortic annulus. In quantitative analysis, especially in strain, the exact location of end systole is important, to identify and measure peak value. In colour imaging, especially in 3D reconstruction, this is not equally important. The post-systolic shortening has a definite duration, extending into the early filling period, where elongation in other areas is visible. The exact location of the time of peak is not important.

\section{Possible applications and further development}

There are two main areas of application. In acute infarction, infarct area is important in prognosis. In addition, identification of an area at risk, may be important in clinical decisions, although the utility of this need to be established.

The other main area is in stress echocardiography. The experienced stress echo cardiographer does a quick visual assessment of the whole cine loop, before scoring in individual segments. This results in quick a decision reached of whether dyssynergy is present or not, and whether segmental scoring is necessary. Colour parametric imaging may to some degree duplicate this by making it possible to evaluate the homogeneity of colour, in one glance, and further to identify the segments where measurement is necessary. Colour display may also facilitate the learning of stress echo interpretation.

\section{Conclusions}

Processing tissue Doppler data from standard apical planes to a four-dimensional data set is quick, and feasible in all patients in this study. The tissue Doppler data can then be quickly post processed to any parameter, to be displayed in bull's eye, M-mode array or 3D surface; each display mode shows different information. Bull's eye displays the whole ventricle, while 3D surface reconstruction gives an approximate representation of true area. Data can also be re extracted from any point as waveforms for quantitative analysis. Parametric display allows quicker identification of both artefacts and dyssynergy than segmental quantitative analysis. Strain rate is best suited to parametric display. The display shows the area of postsystolic shortening in acute infarction exceeds the area of systolic dyssynergy, and may identify an area at risk. Further technical refinements are possible.

\section{Competing interests}

None declared.

\section{Authors' contributions}

HT provided the idea of the specific data format and the programming of the analysis software, $\mathrm{CB}$ did the echocardiography and AS conceived the study design, did the processing, analysis and evaluation of the study. All authors read and approved the final manuscript.

\section{Acknowledgements}

The study was in part supported by a grant from the Norwegian research council.

\section{References}

I. Sutherland GR, Stewart MJ, Groundstroem KW, Moran CM, Fleming A, Guell-Peris FJ, Riemersma RA, Fenn LN, Fox KA and McDicken WN: Color Doppler myocardial imaging: a new technique for the assessment of myocardial function. J Am Soc Echocardiogr 1994, 7:44|-58.

2. Pan C, Hoffmann R, Kuhl H, Severin E, Franke A and Hanrath P: Tissue tracking allows rapid and accurate visual evaluation of left ventricular function. Eur J Echocardiogr 200I, 2:197-202.

3. Höglund $C$, Alam M and Thorstrand C: Atrioventricular valve plane displacement in healthy persons. An echocardiographic study. Acta Med Scand 1988, 224:557-562.

4. Simonson JS and Schiller NB: Descent of the base of the left ventricle: an echocardiographic index of left ventricular function. J Am Soc Echocardiogr 1989, 2:25-35.

5. Alam M, Hoglund $C$ and Thorstrand $C$ : Longitudinal systolic shortening of the left ventricle: an echocardiographic study in subjects with and without preserved global function. Clin Physiol 1992, I 2:443-52.

6. Heimdal A, Stoylen A, Torp $H$ and Skjaerpe T: Real-time strain rate imaging of the left ventricle by ultrasound. J Am Soc Echocardiogr 1998, I 1:1013-19.

7. Greenberg NL, Firstenberg MS, Castro PL, Main M, Travaglini A, Odabashian JA, Drinko JK, Rodriguez LL, Thomas JD and Garcia MJ: Doppler-derived myocardial systolic strain rate is a strong index of left ventricular contractility. Circulation 2002, 105:99-105.

8. Stoylen A, Heimdal A, Bjornstad K, Torp H and Skjaerpe T: Strain rate imaging by ultrasound in the diagnosis of regional dysfunction of the left ventricle. Echocardiography 1999, 16:32I-329.

9. Stoylen A, Heimdal A, Bjornstad K, Wiseth R, Vik-Mo H, Torp H, Angelsen $B$ and Skjaerpe T: Strain rate imaging by ultrasound in the diagnosis of coronary artery disease. J Am Soc Echocardiogr 2000, 13:1053-1064.

10. D'hooge J, Heimdal A, Jamal F, Kukulski T, Bijnens B, Rademakers F, Hatle L, Suetens P and Sutherland GR: Regional Strain Rate Measurements by Cardiac Ultrasound: Principles, Implementation and Limitations. Eur J Echocardiography 2000, I: 154- I 70.

II. Urheim S, Edvardsen T, Torp H, Angelsen B and Smiseth OA: Myocardial strain by Doppler echocardiography. Validation of a new method to quantify regional myocardial function. Circulation 2000, 102: I I58-1 I64.

12. Edvardsen T, Gerber BL, Garot J, Bluemke DA, Lima JA and Smiseth OA: Quantitative assessment of intrinsic regional myocardial deformation by Doppler strain rate echocardiography in humans: validation against three-dimensional tagged magnetic resonance imaging. Circulation 2002, 106:50-56.

13. Brodin LA, van der Linden J and Olstad B: Echocardiographic functional images based on tissue velocity information. Herz 1998 , 23:1183-1199. 
14. Stoylen A, Slordahl S, Skjelvan GK, Heimdal A and Skjaerpe T: Strain Rate Imaging in Normal and Reduced Diastolic Function: Comparison with Pulsed Doppler Tissue Imaging of the Mitral Annulus. J Am Soc Echocardiogr 200 I, I4:264-274.

15. Støylen A, Skjelvan $G$ and Skjaerpe T: Flow propagation velocity is not a simple index of diastolic function in early filling. A comparative study of early diastolic strain rate and strain rate propagation, flow and flow propagation in normal and reduced diastolic function. Cardiovasc Ultrasound 2003, I:3.

16. Bjørnstad K, Maehle J and Aakhus S: Quantitative computerized analysis of left ventricular wall motion. In: Computerized echocardiography Edited by: Domenicucci S, Roelandt J, Pezzano A. Torino: Centro scientifico; I993:4I-55.

17. Maehle J: Assessment of left ventricular volume and regional dysfunction based on 3D endocardial surfaces reconstructed from 2D ultrasound images of the heart. PhD thesis. Norwegian University of Science and Technology, Department of Technical Cybernetics 1996.

18. Andreas Heimdal: Doppler based ultrasound imaging methods for non-invasive assessment of viability. PhD thesis. Norwegian University of Science and Technology, Department of Telematics 1999.

19. Voigt JU, Arnold MF, Karlsson M, Hubbert L, Kukulski T, Hatle L and Sutherland GR: Assessment of Regional Longitudinal Myocardial Strain Rate Derived from Doppler Myocardial Imaging Indexes in Normal and Infarcted Myocardium. J Am Soc Echocardiogr 2000, 13:588-598.

20. Kowalski M, Kukulski T, Jamal F, D'hooge J, Weidemann F, Rademakers F, Bijnens B, Hatle L and Sutherland GR: Can natural strain and strain rate quantify regional myocardial deformation? A study in healthy subjects. Ultrasound in Med \& Biol 200I, 27:1087-1097.

21. Hatle $L$ and Sutherland GR: Regional myocardial function - a new approach. The Grüntzig Lecture. Eur Heart J 2000, 21:1337-1357.

22. Kukulski T, Jamal F, D'Hooge J, Bijnens B, De Scheerder I and Sutherland GR: Acute Changes in Systolic and Diastolic Events During Clinical Coronary Angioplasty: A Comparison of Regional Velocity, Strain Rate, and Strain Measurement. J Am Soc Echocardiogr 2002, 15: I- 12.

23. Wilkenshoff UM, Sovany A, Wigstrom L, Olstad B, Lindstrom L, Engvall J, Janerot-Sjoberg B, Wranne B, Hatle L and Sutherland GR: Regional mean systolic myocardial velocity estimation by real-time color Doppler myocardial imaging: a new technique for quantifying regional systolic function. J Am Soc Echocardiogr 1998, I I:683-692.

24. Schiller NB, Shah PM, Crawford M, DeMaria A, Devereux R, Feigenbaum H, Gutgesell H, Reichek N, Sahn D, Schnittger I, Silverman NH and Tajik J: Recommendations for quantitation of the left ventricle by two-dimensional echocardiography. J Am Soc Echocardiogr 1989, 2:358-367.

Publish with Bio Med Central and every scientist can read your work free of charge

"BioMed Central will be the most significant development for disseminating the results of biomedical research in our lifetime. "

Sir Paul Nurse, Cancer Research UK

Your research papers will be:

- available free of charge to the entire biomedical community

- peer reviewed and published immediately upon acceptance

- cited in PubMed and archived on PubMed Central

- yours - you keep the copyright
BioMedcentral 